\title{
Epilepsy in the Developing World
}

\section{Arturo Carpio, MD, and W. Allen Hauser, MD}

\author{
Corresponding author \\ Arturo Carpio, MD \\ Research Institute, School of Medicine, University of Cuenca, \\ Ciudadela Universitaria, Av. 12 Abril s/n, Cuenca, Ecuador. \\ E-mail: arturo.carpio@ucuenca.edu.ec
}

Current Neurology and Neuroscience Reports 2009, 9:319-326 Current Medicine Group LLC ISSN 1528-4042

Copyright (C) 2009 by Current Medicine Group LLC

Developing countries (DCs) and developed countries have geographic, economic, and social differences. The prevalence and incidence of epilepsy are higher in DCs than in developed countries. However, within DCs, given the high incidence of epilepsy, the prevalence is relatively low, which may be due to high mortality for people with epilepsy (PWE). Neurocysticercosis is one of the main causes of symptomatic epilepsy in many DCs. Prognosis in DCs seems similar to that in developed countries. Because phenobarbital and phenytoin are available and inexpensive, they are the drugs most often used in DCs. The cost of newer antiepileptic drugs and the limited availability of resources for epilepsy care in DCs mean that care for PWE in DCs is marginalized and that many people receive no pharmacologic treatment. The most cost-effective way to decrease the treatment gap in DCs would be to deliver the epilepsy services through primary health care.

\section{Introduction}

There is no established convention for the designation of "developed" and "developing" countries in the United Nation system. The World Bank [1] defines developing countries (DCs) according to the annual gross national product (GNP). Categorization includes low-income countries (GNP per capita up to US \$735), lower-middleincome countries (GNP per capita US \$736-\$2935), and upper-middle-income countries (GNP per capita US \$2936-\$9075). The socioeconomic structure in DCs is marked by profound contrasts based on within-country income. A small percentage of the population has access to institutions (frequently privately owned) that are equipped with the latest and most sophisticated methods of diagnosis, which can offer state-of-the-art service. Most people in DCs suffer the effects of poverty, illiteracy, and malnutrition and must seek health care from a limited number of public institutions that cannot provide optimal services because of economic and administrative limitations. Infectious and parasitic diseases remain among the leading causes of mortality in DCs [2].

Epilepsy is one of the most common serious disorders of the brain, affecting about 50 million people worldwide. It accounts for $1 \%$ of the global burden of disease, $80 \%$ of which is in the developing world, and in some areas $80 \%$ to $90 \%$ of people with epilepsy (PWE) receive no treatment at all [3]. This phenomenon is known as the treatment gap (TG). Major advances in understanding the natural history and social consequences of epilepsy have been made in recent decades, and there have been major improvements in its diagnosis and treatment. There is little information on these topics in DCs, and even when information is available, comparison across studies is difficult because of varying definitions and methodologies [2].

Information regarding available resources for care of PWE is insufficient not only in the developing world but in developed countries. Lack of information concerning existing resources is a major impediment for policymakers at local, national, and international levels trying to plan appropriate services for epilepsy care. On the whole, there are significant inequities within regions and income groups of countries, with low-income countries having meager resources [3].

\section{Prevalence and Incidence}

During the last two decades of the 20th century, there were numerous studies concerning the prevalence of epilepsy in DCs. Lifetime prevalence ranged from 3.7 to 57 per 100,000 person-years [2]. This wide variation was due in part to differences in study methodology. Many studies did not provide clear definitions, and age-adjusted prevalence or age-specific prevalence (a requisite to calculate age-adjusted rates) was not provided. Therefore, it was impossible to compare incidence or prevalence across studies in DCs or with studies in the developed world. Over the past 5 years, there have been several reports regarding the frequency of epilepsy in DCs (Table 1). The reported prevalence is now more consistent because the methodology is frequently based on the recommendations of the International League Against Epilepsy (ILAE) Commission on Epidemiology and Prognosis [2]. The prevalence of active epilepsy in DCs ranges from 3.8 to 15.4 per 1000 person-years (Table 1). The differences are in part related to different study designs and differences regarding the definitions of active epilepsy. Some stud- 


\begin{tabular}{|c|c|c|c|c|c|}
\hline Study & Country & $N$ & $\begin{array}{c}\text { Prevalence, } \\
\quad x 1000\end{array}$ & Data & Study design \\
\hline Wang et al. [4] & China & 58,806 & $4.6^{*}$ & $\begin{array}{c}\text { Treatment gap: } \\
63 \%\end{array}$ & $\begin{array}{l}\text { Door-to-door survey; } \\
\text { community-based }^{+}\end{array}$ \\
\hline $\begin{array}{c}\text { Birbeck and } \\
\text { Kalichi [5] }\end{array}$ & Zambia & 55,000 & $12.5^{* \neq}$ & - & $\begin{array}{l}\text { Door-to-door survey; } \\
\text { community-based }\end{array}$ \\
\hline Del Brutto et al. [11] & Ecuador & 2548 & $10.8^{\S \ddagger}$ & - & $\begin{array}{l}\text { Door-to-door survey; } \\
\text { community-based }\end{array}$ \\
\hline Dent et al. [12] & Tanzania & 141,834 & $7.4^{\S \neq}$ & - & $\begin{array}{l}\text { Door-to-door survey; } \\
\text { community-based }\end{array}$ \\
\hline Medina et al. [13] & Honduras & 7384 & $15.4^{\S}$ & $\begin{array}{c}\text { Incidence: } \\
\text { 92.7/100,000; } \\
\text { treatment gap: } 53 \%\end{array}$ & $\begin{array}{l}\text { Door-to-door survey; } \\
\text { community-based }\end{array}$ \\
\hline Ndoye et al. [6] & Senegal & 480,000 & $14.2^{*}$ & $\begin{array}{l}\text { Treatment gap: } \\
23 \%\end{array}$ & $\begin{array}{l}\text { Door-to-door survey; } \\
\text { community-based }^{+}\end{array}$ \\
\hline Carpio et al. [22] & Ecuador & 420 & - & SMR: 6.3 & Prospective; hospital-based \\
\hline Chen et al. [35] & Taiwan & 263 & - & SMR: 3.7 & Prospective; hospital-based \\
\hline Calisir et al. [14] & Turkey & 2116 & $8.5^{\S \ddagger}$ & - & Community-based \\
\hline Ding et al. [23] & China & 2455 & - & SMR: 3.9 & Prospective; population-based \\
\hline Melcon et al. [10•] & Argentina & 70,000 & $3.8^{\S}$ & - & Community-based \\
\hline Noronha et al. $[9 \bullet \bullet]$ & Brazil & 96,300 & $5.4^{\natural}$ & $\begin{array}{c}\text { Treatment gap: } \\
37.6 \%\end{array}$ & $\begin{array}{l}\text { Door-to-door survey; } \\
\text { community-based }^{+}\end{array}$ \\
\hline $\begin{array}{l}\text { Mungala-Odera } \\
\text { et al. [7] }\end{array}$ & Kenya & 108,896 & $11^{\text {** }}$ & $\begin{array}{l}\text { Incidence: } \\
\text { 187/100,000 }\end{array}$ & $\begin{array}{l}\text { Population-based; > } \\
2 \text { unprovoked seizures } \\
\text { in children }\end{array}$ \\
\hline Tran et al. [15] & Laos & 23,788 & $7.7^{\S}$ & - & $\begin{array}{l}\text { Door-to-door survey; } \\
\text { community-based }\end{array}$ \\
\hline Bielen et al. [17•] & Croatia & 212,069 & $5.0^{\S \ddagger}$ & - & Health institution-based \\
\hline Kaiser et al. [24] & Uganda & 61 & - & SMR: 7.2 & Prospective; population-based \\
\hline Edwards et al. $[8 \bullet \bullet]$ & Kenya & 253,291 & $4.5^{* \neq}$ & $\begin{array}{c}\text { Treatment gap: } \\
70.3 \%\end{array}$ & $\begin{array}{l}\text { Community-based; children } \\
<6 \text { y were excluded }\end{array}$ \\
\hline Tuan et al. [16•] & Vietnam & 47,269 & $4.4^{\S \neq}$ & - & $\begin{array}{c}\text { Door-to-door survey; } \\
\text { community-based }^{+}\end{array}$ \\
\hline \multicolumn{6}{|c|}{$\begin{array}{l}\text { *Active epilepsy was defined as at least two unprovoked seizures in the preceding year. } \\
\text { TThese studies were part of the Global Campaign Against Epilepsy demonstration projects supported by the International League Against } \\
\text { Epilepsy, International Bureau for Epilepsy, and World Health Organization. } \\
\text { *Age-adjusted. } \\
\text { sActive epilepsy was defined as at least two unprovoked seizures in the preceding } 5 \text { years. } \\
\text { 'Active epilepsy was defined as at least two unprovoked seizures in the preceding } 2 \text { years. } \\
\text { SMR-standardized mortality ratio. }\end{array}$} \\
\hline
\end{tabular}

ies require two or more unprovoked seizures in the year before the index date to be considered "active epilepsy" $[4-5,8 \bullet \bullet]$; others use a 2-year period [9] or 5-year period $[10 \bullet, 11-15,16 \bullet, 17 \bullet]$. Limiting assessment to studies that provide information for age-adjusted rates (Table 1), the average age-adjusted prevalence of active epilepsy is 8.5 per 1000 people. A Brazilian study reported a higher prevalence of epilepsy in more deprived social classes

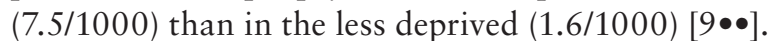

Incidence studies require surveillance of a population of adequate size over a sufficiently long period of time to assure stable estimates of frequency. As a result, incidence studies are expensive and relatively few reports are available for total pop- ulations. In developed countries, the age-adjusted incidence of epilepsy ranges from 24 to 53 per 100,000 [18]. In DCs, based on two studies carried out in South America in the past decade, incidence ranged from 114 to 190 per 100,000 [2], which is considerably higher than that reported in industrialized countries. Recent studies confirm the high incidence: a hospital-based study of children in Tunisia reported the incidence of first unprovoked seizures to be 102 per 100,000 [19]. A population-based study carried out among children in Kenya reported the incidence of epilepsy (two or more unprovoked seizures) to be 187 per 100,000 [8••]. In a population-based study among all ages in Honduras [14], the incidence of nonfebrile seizures was 92.7 per 100,000 (Table 1). Because of 
the broader case-inclusion criteria and uncertainty regarding age-specific distribution in these incidence studies, there is no way to compare the results.

Studies from developed countries show a consistent pattern suggesting that the onset of epilepsy occurs at both extremes of life [18]. However, the incidence in DCs is highest among young and middle-aged adults [2], perhaps as a manifestation of prevalent diseases in these age groups, including conditions such as the parasitic diseases that are endemic in these countries and brain injury infections.

A recent population-based study was performed to determine the incidence of first unprovoked seizures or newly diagnosed epilepsy in a low-income, predominantly Hispanic population in Northern Manhattan, New York City $[20 \bullet \bullet$. Lower income was associated with higher incidence $(164 / 100,000)$. Interestingly, this incidence is similar to those reported in comparable populations of DCs.

\section{Prognosis}

The prognosis of epilepsy can be measured in several ways: according to the risk of recurrence after a seizure, probability of remission, risk of relapse after drug withdrawal, and mortality in PWE [21]. In DCs, the prognosis of epilepsy might be different from that in industrialized countries, but few studies of natural history allow comparisons [2]. Methodologic shortcomings also contribute to the uncertainties.

Around the world, the prognosis of epilepsy is favorable for most PWE. Mortality in PWE is increased in DCs as well as developed countries [22-24]. The absolute mortality is higher in DCs, which may in part explain why prevalence tends to be only slightly higher $(8.5 / 1000)$ than in developed countries. In contrast, the incidence of epilepsy is two to three times higher than in industrialized countries. Because increased mortality only accounts for a small portion of the deficit of cases, the most likely explanation for the similarity of the prevalence rates is spontaneous remission in many people [21].

About $70 \%$ of PWE achieve long-term remissionmost within 5 years of diagnosis [21]. In DCs, after a first unprovoked seizure, the risk of recurrence is $33 \%$ to $37 \%$, which is similar to that among developed countries [25-27]. Studies from DCs show that epilepsy secondary to underlying structural causes [26] or with an abnormal electroencephalogram confers the worst prognosis [27]. The type of seizure (generalized or partial) and the drug treatment used seem not to affect prognosis. Calcifications on CT suggest a probable parasitic or infectious etiology and do not increase the risk of recurrence $[28,29]$. No studies of the prognosis of epileptic syndromes have been published from DCs.

\section{Prognosis for seizure recurrence in patients with neurocysticercosis}

Neurocysticercosis (NC) is endemic in some DCs but is now becoming a serious health problem in industrialized nations with high immigrant populations. Acute symptomatic seizures are the main clinical manifestation of NC [28]. Based on prospective studies, NC generally has an overall favorable prognosis in terms of seizure control and seizure remission [28,29]. A Brazilian study confirms the good prognosis of parenchymal NC [29]. The authors point out that inflammatory processes related to parasitic infection, death, degeneration, and calcifications in the brain of the host do not significantly aggravate the cognitive deficits observed in medically refractory mesial temporal lobe epilepsy.

A clinical trial regarding treatment with cysticidal drugs for patients with epilepsy due to NC did not find a reduction in the number of seizures overall but did report a reduction in the number of generalized seizures in patients treated with albendazole [30]. Recent clinical trials have shown that treatment with cysticidal drugs does not modify the long-term seizure outcome in patients with NC $[31 \bullet \bullet, 32,33 \bullet \bullet]$. Even worse, cysticidal therapy may have an adverse effect with respect to seizure control, encephalopathy, recurrent hospital admissions, calcification of lesions, and cost of treatment [34•].

Interpreting the risk of seizures after NC is difficult because of the failure to distinguish acute symptomatic seizures from epilepsy. These difficulties are increased in patients who have mixed forms, including active, transitional, and calcified lesions. Further studies should be performed to estimate recurrence risk in patients with probable unprovoked seizures with or without calcifications but without other lesions versus patients with acute seizures due to transitional cysts.

\section{Mortality}

In developed countries, the overall mortality associated with epilepsy is two to three times greater than that found in the general population [21]. In DCs, it is almost impossible to ascertain the number of deaths due to epilepsy because incidence studies are difficult to conduct, death certificates are unreliable, autopsies are not easy to obtain, and the cause of death is not usually known with certainty [2]. There are few reliable coroners' reports, and many of the reports that are available are incomplete. Migration makes large, prospective, population-based mortality studies of epilepsy difficult to carry out $[22,35]$.

Few studies of mortality in PWE have been performed in DCs. It seems that mortality in PWE in these countries is generally higher than that reported in developed countries. The standardized mortality ratio (calculated by comparing the observed number of deaths in the epilepsy cohort with that expected in the general population) ranges from 3.7 [35] and 3.9 [23] in prospective, cohort, population-based studies in China to 6.3 in a prospective cohort study in Ecuador [22] and 7.2 in a populationbased study in Uganda [24]. These ratios are higher than the twofold to threefold increases reported in developed countries. It is not possible to generalize these mortality 
numbers to the general population because they were obtained from selected populations. Prospective studies are needed in DCs to follow a large cohort with newonset epilepsy for a defined length of time to determine the mortality. These studies are expensive and difficult to perform, but they are necessary if we are to understand the increase of mortality in epilepsy in DCs.

\section{Etiology}

In DCs, analytical epidemiologic studies designed to establish associations and determinants of epilepsy have been scarce. Comparing results of studies of etiology is difficult because of differences in definitions and lack of diagnostic criteria [36]. Most studies lack information on the latency between the first acute symptomatic seizure (if any) and the first unprovoked seizure, age at onset of seizures, and age at diagnosis. In studying etiology, it is preferable to use incident cases instead of prevalent cases because with prevalent cases one cannot distinguish the potential etiologic factors that preceded the onset of epilepsy from those that occurred after the disease developed. Cause and effect become confused. Despite the lack of uniform criteria, newly available information shows that the rates of idiopathic/cryptogenic epilepsy $(60 \%-70 \%)$ and symptomatic epilepsy $(30 \%-40 \%)$ remain constant both in developing and developed countries [2].

Etiology and risk factors for epilepsy vary with age and geographic location. Congenital, developmental, and genetic conditions are mostly associated with epilepsy in childhood, adolescence, and early adulthood. Head trauma, central nervous system infections, and tumors may occur at any age and may lead to epilepsy, but tumors are more common after age 40 . Cerebrovascular disease is the most common risk factor for epilepsy in people older than 60. In some parts of DCs, endemic infections such as malaria, NC, paragonimiasis, and toxocariasis are associated with epilepsy $[36,37,38 \bullet \bullet]$.

Studies of highly selected patients with seizures in neurologic services of hospital settings from some DCs reported NC to be the main cause of epilepsy, accounting for $30 \%$ to $50 \%$ of cases $[11,13]$. It is likely that most people with NC have acute symptomatic seizures that do not necessarily evolve into epilepsy. This is one of the reasons that epilepsy is overdiagnosed in some studies in which there is no differentiation between seizures and epilepsy [36]. In about two-thirds of patients with acute symptomatic seizures due to NC, the seizures do not recur [28].

In a recent study of 212 PWE in a rural sub-Saharan Africa area endemic for porcine cysticercosis using CT to facilitate the diagnoses, $2.4 \%$ were identified as having definitive $\mathrm{NC}$ and $11 \%$ had lesions highly suggestive

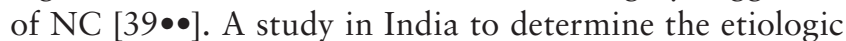
role of NC in a hospital-based sample in 1026 patients with epilepsies divided results according to the ILAE's epidemiologic criteria [36]. NC was diagnosed on imaging studies in $59 \%$ of those with a single seizure but only
$2.0 \%$ with prevalent epilepsy. These results confirm the necessity of differentiating between acute seizure and recurrent unprovoked seizures (epilepsy).

A study in rural Ecuador concluded that NC is associated with one in three cases of epilepsy and was possibly the cause of the excessive amount of epilepsy in that population [11]. However, only three of 24 PWE (8\%) had "definitive" NC. A community survey in Peru showed that seroprevalence (using the enzyme-linked immunoelectrotransfer blot [EITB] assay) was positive in $24.2 \%$ (200/825); $38.5 \%$ of individuals (15/39) with seizures in this community had lesions compatible with NC on CT [40]. The authors concluded that brain CT abnormalities compatible with $\mathrm{NC}$ were more frequent in individuals with seizures and in those with a positive EITB assay for cysticercosis. Most of the patients who were diagnosed with NC had only calcifications (half of them had just one calcification). However, brain calcifications are not synonymous with NC, and the small number of people in these studies do not allow generalizations. Some authors have reported an association between seizures and NC based on positive serum antibodies to Taenia solium/cysticercosis [41]; unfortunately, the presence of antibodies may indicate only previous exposure to or infection with the parasite but not necessarily a brain infection.

$\mathrm{NC}$ and epilepsy are common in most DCs. Because of the high prevalence of both conditions, some of the coexistence of the two conditions could occur by chance alone [29]. New interesting studies have suggested that perilesional edema is associated with episodic seizure activity in patients with calcified NC [42•], but it is not clear whether this edema is causal or a consequence of the seizure. An irritative zone in the temporal lobe is more relevant in determining the severity, symptomatology, and frequency of seizures than the number and location of calcifications [43•]. These findings provide a better understanding of the relationship between $\mathrm{NC}$ and epilepsy.

\section{Treatment}

The choice of drug in most DCs depends on availability and cost. Phenobarbital (PB) and phenytoin, which are commonly available and inexpensive, are the drugs that are used most often in DCs [2]. Widely available but more expensive choices include carbamazepine and sodium valproate. The administration of all of these antiepileptic drugs (AEDs) entails additional problems in DCs aside from side effects. Anemia and hepatitis are already associated problems in DCs; therefore, it may be impossible to differentiate problems of AEDs from concurrent illnesses. Malabsorption syndromes, malnutrition with hypoproteinemia, and hepatic dysfunction may interfere with the pharmacokinetics of AEDs [44].

Epilepsy is a controllable disorder in most people. About $60 \%$ to $70 \%$ of patients will become seizure free with AED therapy. Treatment does not need to be expensive. The direct drug cost of treating a patient for a year 
with PB could be as low as US $\$ 2.60$ [45]. The World Health Organization (WHO) recommends $\mathrm{PB}$ as the treatment of choice for partial and tonic-clonic seizures in resource-restricted countries [3]. This policy has been questioned in the developed world, because PB is thought to be less well tolerated than other AEDs [45]. Since PWE in developed countries are seldom prescribed PB because of its toxicity, some feel it would be unethical to recommend its use in DCs. Nonetheless, if the drug is not as toxic as it is believed to be, it might be used more widely in the developed world [46]. PB has many favorable features: broad-spectrum efficacy against all seizure types other than absences; seizure freedom rates comparable to those associated with modern drugs; long half-life compatible with once-daily dosing; and, especially, low cost. Disadvantages include enzyme induction, sedation, adverse cognitive and behavioral effects (particularly in children), and risk of fetal malformations in offspring of PWE [46].

Controlled trials in DCs suggest that few differences in efficacy exist between PB and other established AEDs. Most studies in DCs do not show excess neuropsychological toxicity of $\mathrm{PB}$ compared with other anticonvulsants, possibly because dosages in these studies tended to be lower than those used in developed countries or because lack of options make people less willing to report side effects [45]. A study of children in Bangladesh [47•] found no significant difference in behavioral problems such as restlessness and hyperactivity between $\mathrm{PB}$ and carbamazepine ( $7 \%$ vs $11 \%$ ) and no significant difference in psychological and behavioral assessments after 1 year. Among children who completed a 1-year follow-up, $47.5 \%$ of those on $\mathrm{PB}$ and $60 \%$ of those on carbamazepine were seizure free for the most recent 6 months. Nonetheless, this study has limitations, including an open-label design and low power to detect potentially important differences in seizure outcome and behavioral test scores. A large community-based intervention study at the primary care level in rural China enrolled 2455 patients with convulsive epilepsy [48•]. Physicians with basic training could treat epilepsy patients with $\mathrm{PB}$, with beneficial effects for most patients with convulsive seizures. Few cognitive or behavioral adverse events were noted, but formal psychometric testing was not done.

Newer AEDs, including oxcarbazepine, lamotrigine, gabapentin, topiramate, levetiracetam, and pregabalin, are available, locally manufactured, and sold in very few DCs with a developed pharmaceutical industry. However, because many of these drugs are still unaffordable to most people in DCs, the clinician should always consider cost before prescribing them [2].

\section{Treatment gap}

According to the WHO, about $80 \%$ of the 35 million PWE who live in DCs receive no treatment at all [3]. As a consequence, they experience morbidity related to seizures and the psychosocial consequences of stigma and discrimi- nation. Unfortunately, most of these people-many of whom are children-could have their seizures completely controlled and return to a normal life by taking a single daily dose of a drug that costs less than $\$ 3(£ 1.50$; $€ 2.20)$ each year [45].

The TG is defined as the number of people with active epilepsy not on treatment or on inadequate treatment; it is expressed as a percentage of the total number with active epilepsy [49]. The gap is reported to be influenced by various factors, including lack of access to or knowledge of AEDs, poverty, cultural beliefs, stigma, poor health delivery infrastructure, and shortage of trained professionals [49]. A wide variation of the TG has been reported over the past 5 years $[5,7,9 \bullet \bullet, 11,14]$, ranging from $23 \%$ to $70 \%$ (Table 1 ). In a systematic review of the TG in DCs, Mbuba et al. [50 • $]$ observed a high degree of heterogeneity and inconsistency between studies. The overall estimate of the TG was $56 \%$, which is much lower than the $80 \%$ to $90 \%$ that is widely quoted in many studies $[3,49]$. The variation in estimates may be explained by nonuniform methods used to estimate the TG as well as the diverse study populations. The TG was mainly attributed to inadequate skilled manpower, cost of treatment, cultural beliefs, and unavailability of AEDs.

In addition to the TG, a wide gap exists between the need for AEDs and their availability. Only $20 \%$ of the potential market for AEDs is in developed countries, but these countries receive more than $80 \%$ of the total global supply of AEDs. This means that DCs receive only $10 \%$ to $20 \%$ of AEDs, but they have a greater need for them [51].

\section{Surgical treatment}

Surgery for epilepsy has been proved feasible and costeffective in DCs [52•]. However, it is available in only $13 \%$ of low-income countries, versus $66 \%$ of high-income countries [3]. The success of epilepsy surgery depends on the accurate identification of good surgical candidates and the selection of the best candidates based on the available resources and technologies without endangering safety. Selecting patients amenable to surgery with the use of limited resources is a key issue in DCs. In this regard, training physicians capable of compiling a good history and referring appropriate patients to the epileptologist is critical [52•]. Most patients are seen and followed at primary and secondary care facilities, where the attending physicians may have limited knowledge of current concepts in managing epilepsies. As an important step in the care of medically refractory patients with epilepsy, appropriate primary health care strategies should be defined and introduced in DCs to educate physicians on the diagnosis of epilepsy and various management strategies.

Nowadays, there is increasing enthusiasm for developing epilepsy surgery programs in DCs. For example, in Uganda, a facility was developed for the treatment of intractable temporal lobe epilepsy that functions within the technologic and expertise constraints of a severely low-resource area [53•]. This program relies on partner- 
ship with epilepsy professionals and training of local staff. The aim of the program was to evaluate the feasibility of an epilepsy surgery program in a resource-limited setting. The investigators used technology and expertise that was reasonably available and could function in a sustained manner in this setting.

\section{Care}

The Global Campaign Against Epilepsy: Out of the Shadows program was launched in 1997 "to improve acceptability, treatment, services and prevention of epilepsy worldwide" [3]. The campaign is conducted by the WHO in partnership with the ILAE and the International Bureau for Epilepsy (IBE). The aim of the campaign is principally to reduce the TG by providing better information about epilepsy and its consequences and to help governments and those concerned with epilepsy to reduce the burden of the disorder.

Many constraints and difficulties hinder the provision of adequate epilepsy care in DCs. Some of the major stumbling blocks include poor community knowledge and awareness, cultural beliefs, stigma, poor economy, and the lack of drug supplies (due to logistics or the economy), infrastructure, and government resources [2]. Worldwide, there are large inequities in the cost of first-line AEDs and the availability of resources for epilepsy care, with low-income countries having extremely limited resources. There is also inequity in the availability of diagnostic services in DCs. Although most new technologies are available in some DCs, they are often concentrated in only a few centers or major cities $[2,3]$.

The most cost-effective way to decrease the TG in DCs would be to deliver epilepsy services through primary health care. DCs often lack personnel with neurologic expertise who can recognize and appropriately treat epilepsy patients [54]. The ideal neurologist-to-population ratio is $1: 100,000$, and this ratio is not achieved in most DCs. The main tasks of the personnel in primary care settings involved in epilepsy care should include follow-up and monitoring of treatment, maintenance and/or prescription of AEDs, information and education of patients and caregivers, referral of PWE for specialist treatment, and case ascertainment [55]. Where resources are available, the care of epilepsy patients should be provided by a multidisciplinary team. Through more appropriate and timely use of these treatment modalities and better organization in the delivery of care, it is hoped that more PWE around the world can benefit from effective treatment and live safer and more fulfilling lives [3,4].

One of the main activities of the ILAE/IBE/WHOdesigned to help countries develop national programs on epilepsy-envisioned the initiation and implementation of demonstration projects with the ultimate goal of developing a variety of successful models of epilepsy control that could be integrated into the participating countries' health care systems $[3,51]$. Demonstration projects have been carried in China [4], Togo [56], Brazil [9], Senegal [6], and Vietnam [17•].

From the epidemiologic perspective, epilepsy is a significant cause of disability and disease burden in the world. Disability-adjusted life years (DALYs) have been proposed as an indicator of the health effects of health care systems and are used to evaluate the impact of mortality and morbidity together [57]. Ding et al. [58] carried out a study to demonstrate the application of DALYs as an aid in health outcome measures to evaluate the epilepsy disease burden. The authors reported that the DALYs lost because of epilepsy was 2.08 per 1000 people, representing the epilepsy disease burden in rural China.

\section{Conclusions}

The studies examined in this article lead to a number of conclusions.

- The prevalence of epilepsy in DCs is about $8.5 / 1000$, which is similar to the rate in developed countries. The prevalence is low given the high incidence of epilepsy in DCs and may be due to the fact that mortality of PWE is higher than that of developed countries.

- The incidence of epilepsy in DCs (more than $92 / 100,000)$ is higher, mainly in children, than in developed countries (40/100,000 to 70/100,000) and is probably related to a high prevalence of risk factors in poor countries, such as poor perinatal care, a higher frequency of head injury, and a high prevalence of infectious and parasitic diseases.

- The rates of idiopathic/cryptogenic (60\%-70\%) and symptomatic $(30 \%-40 \%)$ epilepsy in DCs are similar in developed countries. However, the causes of epilepsy in the symptomatic group in DCs are different than in developed countries.

- It seems that the mortality rate from epilepsy is much higher in DCs than in industrialized countries.

- The risk of seizure recurrence in people with newly diagnosed epilepsy is similar in DCs and industrialized countries. There is an increased risk of early seizure recurrence in patients with symptomatic epilepsy and patients with an abnormal CT scan. These results are also comparable to those reported in industrialized nations.

- To establish risk factors and mortality for epilepsy in DCs, further prospective analytical studies are needed. New epidemiologic measures such as DALYs should be used to evaluate the epilepsy disease burden.

- Concerted multidisciplinary efforts concentrating on the risk factors (eg, enforcement of strict traffic regulations and improvement in perinatal care) or the provision of specific protection (eg, vaccination) for preventable causes can help to decrease substantially the burden attributable to epilepsy. 
- $\quad \mathrm{PB}$ may be less toxic than generally thought and might be considered more often to treat PWE in DCs.

- The most cost-effective way to decrease the TG in most countries would be to deliver epilepsy services through primary health care. Future research should estimate the TG coherently and develop sustainable interventions that will address the causes.

- The ILAE/IBE/WHO should pressure pharmaceutical companies to reduce the cost of new AEDs in low-income countries. These organizations should make a major effort to bring new AEDs to low-income countries. Widespread epilepsy treatment will be an extremely sound global investment toward the economic, social, and political wellbeing of the world's poor.

\section{Disclosure}

No potential conflicts of interest relevant to this article were reported.

\section{References and Recommended Reading}

Papers of particular interest, published recently, have been highlighted as:

- Of importance

$\bullet \quad$ Of major importance

1. The World Bank: Global Economic Prospects 2004: Realizing the Development Promise of the Doha Agenda. Available at http://siteresources.worldbank.org/ INTRGEP2004/Resources/gep2004fulltext.pdf. Accessed May 21, 2009.

2. Bharucha NE, Carpio A, Gallo Diop A: Epidemiology of epilepsy in developing countries. In Epilepsy: A Comprehensive Textbook, edn 2. Edited by Engel P, Pedley T. Philadelphia: Lippincott Williams \& Wilkins; 2008:89-101.

3. World Health Organization: Atlas: Epilepsy Care in the World. Geneva: World Health Organization; 2005.

4. Wang WZ, Wu JZ, Wang DS, et al.: The prevalence and treatment gap in epilepsy in China: an ILAE/IBE/WHO study. Neurology 2003, 60:1544-1545.

5. Birbeck GL, Kalichi EM: Epilepsy prevalence in rural Zambia: a door-to-door survey. Trop Med Int Health 2004, 9:92-95.

6. Ndoye NF, Sow AD, Diop AG, et al.: Prevalence of epilepsy its treatment gap and knowledge, attitude and practice of its population in sub-urban Senegal an ILAE/IBE/WHO study. Seizure 2005, 14:106-111.

7. Mungala-Odera V, White S, Meehan R, et al.: Prevalence, incidence and risk factors of epilepsy in older children in rural Kenya. Seizure 2008, 17:396-404.

8.• Edwards T, Scott AG, Munyoki G, et al.: Active convulsive epilepsy in a rural district of Kenya: a study of prevalence and possible risk factors. Lancet Neurol 2008, 7:50-56.

This large community survey of epilepsy in a rural Kenyan district reports not only on prevalence but the TG and potentially preventable risk factors for epilepsy.

9.• Noronha AL, Borges MA, Marques LH, et al.: Prevalence and pattern of epilepsy treatment in different socioeconomic classes in Brazil. Epilepsia 2007, 48:880-885.

This study is the first door-to-door epidemiologic survey of epilepsy, the TG, and the socioeconomic influence on epilepsy in a general Brazilian population.
10. Melcon MO, Kochen S, Vergara RH: Prevalence and clinical features of epilepsy in Argentina. A community-based study. Neuroepidemiology 2007, 28:8-15.

This study is one of the first community-based studies of the prevalence of active epilepsy in Argentina, based on ILAE's definitions.

11. Del Brutto OH, Santibanez R, Idrovo L, et al.: Epilepsy and neurocysticercosis in Atahualpa: a door-to-door survey in rural coastal Ecuador. Epilepsia 2005, 46:583-587.

12. Dent W, Helbok R, Matuja WB, et al.: Prevalence of active epilepsy in a rural area in South Tanzania: a door-to-door survey. Epilepsia 2005, 46:1963-1969.

13. Medina MT, Duron RM, Martinez L, et al.: Prevalence, incidence, and etiology of epilepsies in rural Honduras: the Salama study. Epilepsia 2005, 46:124-131.

14. Calisir N, Bora I, Irgil E, Boz M: Prevalence of epilepsy in Bursa city center, an urban area of Turkey. Epilepsia 2006, 47:1691-1699.

15. Tran DS, Odermatt P, Le TO, et al.: Prevalence of epilepsy in a rural district of central of Lao PDR. Neuroepidemiology 2006, 26:199-206.

16. Tuan NA, Cuong le Q, Allebeck P, et al.: The prevalence of epilepsy in a rural district of Vietnam: a populationbased study from the EPIBAVI project. Epilepsia 2008, 49:1634-1637.

This population-based study of epilepsy is from a representative rural region of Vietnam.

17. Bielen I, Cvitanovic-Sojat L, Bergman-Markovic B, et al.: Prevalence of epilepsy in Croatia: a population-based survey. Acta Neurol Scand 2007, 116:361-367.

This study was carried out by primary health care physicians and neurologists to determine the prevalence of active epilepsy in Croatia; the results are in keeping with findings from other European countries.

18. Banerjee PN, Hauser WA: Incidence and prevalence. In Epilepsy: A Comprehensive Textbook, edn 2. Edited by Engel P, Pedley T. Philadelphia: Lippincott Williams \& Wilkins; 2008:45-56.

19. Dogui M, Jallon P, Tamallah JB, et al.: Episousse: incidence of newly presenting seizures in children in the Region of Sousse, Tunisia. Epilepsia 2003, 44:1441-1444.

20.• Benn EK, Hauser WA, Shih T, et al.: Estimating the incidence of first unprovoked seizure and newly diagnosed epilepsy in the low-income urban community of Northern Manhattan, New York City. Epilepsia 2008, 49:1431-1439.

This interesting study suggests that the incidence of newly

diagnosed epilepsy does not differ by race or ethnicity, but lower income is associated with a higher incidence.

21. Beghi E, Sander JW: The natural history and prognosis of epilepsy. In Epilepsy: A Comprehensive Textbook, edn 2. Edited by Engel P, Pedley T. Philadelphia: Lippincott Williams \& Wilkins; 2008:65-70.

22. Carpio A, Bharucha NE, Jallon P, et al.: Mortality of epilepsy in developing countries. Epilepsia 2005, 46(Suppl 11):28-32.

23. Ding D, Wang W, Wu J, et al.: Premature mortality in people with epilepsy in rural China: a prospective study. Lancet Neurol 2006, 5:823-827.

24. Kaiser C, Asaba G, Kasoro S, et al.: Mortality from epilepsy in an onchocerciasis-endemic area in West Uganda. Trans R Soc Trop Med Hyg 2007, 101:48-55.

25. Daoud AS, Ajloni S, El-Salem K, et al.: Risk of seizure recurrence after a first unprovoked seizure: a prospective study among Jordanian children. Seizure 2004, 13:99-103.

26. Kochen S, Melcon MO: Prognosis of epilepsy in a community-based study: 8 years of follow-up in an Argentine community. Acta Neurol Scand 2005, 112:370-374.

27. Scotoni AE, Manreza ML, Guerreiro MM: Recurrence after a first unprovoked cryptogenic/idiopathic seizure in children: a prospective study from Sao Paulo, Brazil. Epilepsia 2004, 45:166-170.

28. Carpio A, Hauser W: Prognosis for seizure recurrence in patients with newly diagnosed neurocysticercosis. Neurology 2002, 59:1730-1734. 
29. Terra-Bustamante VC, Coimbra ER, Rezek KO, et al.: Cognitive performance of patients with mesial temporal lobe epilepsy and incidental calcified neurocysticercosis. $J$ Neurol Neurosurg Psychiatry 2005, 76:1080-1083.

30. Garcia H, Pretell E, Gilman R, et al.: A trial of antiparasitic treatment to reduce the rate of seizures due to cerebral cysticercosis. N Engl J Med 2004, 350:249-258.

31.• De Souza A, Thennarasu K, Yeshraj G, et al.: Randomized controlled trial of albendazole in new onset epilepsy and MRI confirmed solitary cerebral cysticercal lesion: effect on long-term seizure outcome. J Neurol Sci 2009 , 276:108-114.

This interesting prospective randomized controlled study shows that albendazole does not alter the long-term seizure outcome in patients with solitary cerebral cysticercal lesion and epilepsy.

32. Thussu A, Chattopadhyay A, Sawhney IM, Khandelwal N: Albendazole therapy for single small enhancing CT lesions (SSECTL) in the brain in epilepsy. I Neurol Neurosurg Psychiatry 2008, 79:272-275.

33.• Carpio A, Kelvin E, Bagiella E, et al.: The effects of albendazole treatment on neurocysticercosis: a randomized controlled trial. I Neurol Neurosurg Psychiatry 2008, 79:1050-1055.

This double-blind randomized placebo-controlled clinical trial demonstrates that albendazole therapy does not alter the long-term seizure outcome in patients with epilepsy.

34. Das K, Mondal GP, Banerjee M, et al.: Role of antiparasitic therapy for seizures and resolution of lesions in neurocysticercosis patients: an 8 year randomised study. J Clin Neurosci 2007, 14:1172-1177.

This randomized controlled study was designed to evaluate albendazole therapy in patients with NC, who were prospectively followed for more than 5 years.

35. Chen RC, Chang YC, Chen TH, et al.: Mortality in adult patients with epilepsy in Taiwan. Epileptic Disord 2005 , 7:215-219

36. Carpio A, Hauser WA: Remote symptomatic epilepsies and epileptic syndromes: etiological spectrum. In Epilepsy in the Tropics. Edited by Murthy JMK, Senanayke N. Georgetown, TX: Landes Bioscience; 2006:72-84.

37. Carter JA, Neville BG, White $S$, et al.: Increased prevalence of epilepsy associated with severe falciparum malaria in children. Epilepsia 2004, 45:978-981.

38.• Singh G, Singh P, Singh I, et al.: Epidemiologic classification of seizures associated with neurocysticercosis: observations from a sample of seizure disorders in neurologic care in India. Acta Neurol Scand 2006, 113:233-240.

This interesting study suggests that NC is most commonly identified in individuals with single seizures, but it is rarely is associated with recurrent unprovoked seizures (or epilepsy).

39.• Winkler AS, Blocher J, Auer H, et al.: Anticysticercal and antitoxocaral antibodies in people with epilepsy in rural Tanzania. Trans R Soc Trop Med Hyg 2008, 102:1032-1038.

This is the first study in sub-Saharan Africa to report evidence within a large-scale neuroimaging assessment that $\mathrm{NC}$ is a main cause of epilepsy.

40. Montano SM, Villaran MV, Ylquimiche L, et al.: Neurocysticercosis: association between seizures, serology, and brain CT in rural Peru. Neurology 2005, 65:229-223.

41. Prasad KN, Prasad A, Gupta RK, et al.: Neurocysticercosis in patients with active epilepsy from the pig farming community of Lucknow district, north India. Trans $R$ Soc Trop Med Hyg 2009, 103:144-150.

42.• Nash TE, Pretell EJ, Lescano AG, et al.: Perilesional brain oedema and seizure activity in patients with calcified neurocysticercosis: a prospective cohort and nested case-control study. Lancet Neurol 2008, 7:1099-1105.

This study suggests that perilesional edema on imaging studies is common and associated with episodic seizure activity in patients with calcified NC.
43. Kowacs PA, Rogacheski E, Muzzio J, Werneck LC: The role of the irritative zone and of the number and distribution of calcifications in the severity of epilepsy associated with intracranial calcifications. Arq Neuropsiquiatr 2006 64:905-911.

This study found no relationship between the number of calcifications and epilepsy severity.

44. Pack AM, Morrell MJ, Marcus R, et al.: Bone mass and turnover in women with epilepsy on antiepileptic drug monotherapy. Ann Neurol 2005, 57:252-257.

45. Perucca E: Treatment of epilepsy in DC. BMJ 2007, 334:1175-1176.

46. Kwan P, Brodie MJ: Phenobarbital for the treatment of epilepsy in the 21st century: a critical review. Epilepsia 2004, 45:1141-9114.

47. Banu SH, Jahan M, Koli UK, et al.: Side effects of phenobarbital and carbamazepine in childhood epilepsy: randomized controlled trial. BMJ 2007, 334:1207.

This study found no significant difference in behavioral problems between PB and carbamazepine and no significant difference in psychological and behavioral assessments after 1 year of follow-up.

48.• Wang WZ, Wu JZ, Ma GY, et al.: Efficacy assessment of phenobarbital in epilepsy: a large, community-based intervention trial in rural China. Lancet Neurol 2006, 5:46-52. This pragmatic study shows that physicians with basic training could treat epilepsy patients with $\mathrm{PB}$, with beneficial effects for most patients with seizures. Few cognitive or behavioural adverse events were noted, but formal psychometric testing was not done.

49. Meinardi H, Scott RA, Reis R, Sander JW: The treatment gap in epilepsy: the current situation and ways forward. Epilepsia 2001, 42:136-149.

50.• Mbuba CK, Ngugi AK, Newton CR, Carter JA. The epilepsy treatment gap in DC: a systematic review of the magnitude, causes, and intervention strategies. Epilepsia 2008, 49:1491-1503.

The authors present a complete review and a meta-analysis of the TG in epilepsy in developing countries.

51. Sander JW: Global campaign against epilepsy. Overview of the demonstration projects. Epilepsia 2002, 43(Suppl 6):34-36.

52. Asadi-Pooya AA, Sperling MR: Strategies for surgical treatment of epilepsies in DC. Epilepsia 2008, 49:381-385.

This review suggests that epilepsy surgery has been proved to be efficacious, safe, and cost-effective in DCs.

53.• Boling W, Palade A, Wabulya A, et al.: Surgery for pharmacoresistant epilepsy in the developing world: a pilot study. Epilepsia 2009 Jan 19 (Epub ahead of print).

This study proposes a model for surgical treatment of pharmacoresistant temporal lobe epilepsy for use in low-resource settings in the developing world.

54. Murthy JM: Some problems and pitfalls in developing countries. Epilepsia 2003, 44(Suppl 1):38-42.

55. Carpio A, Lisanti N, Calle H, et al.: Validation of a questionnaire for epilepsy diagnosis in primary care services. Rev Panam Salud Publica 2006, 19:157-162.

56. Balogou AA, Grunitzky EK, Belo M, et al.: Management of epilepsy patients in Batamariba district, Togo. Acta Neurol Scand 2007, 116:211-216.

57. Begley CE, Baker GA, Beghi E, et al.: Cross-country measures for monitoring epilepsy care. Epilepsia 2007, 48:990-1001.

58. Ding D, Hong Z, Wang WZ, et al.: Assessing the disease burden due to epilepsy by disability adjusted life year in rural China. Epilepsia 2006, 47:2032-2037. 